\title{
Un modèle cinématique discret de déformation 3D d'un bassin sédimentaire
}

\author{
T. Cornu' , F. Schneider ${ }^{1}$ et J.P. Gratier ${ }^{2}$ \\ 1 Institut français du pétrole, 1 et 4, avenue de Bois-Préau, 92852 Rueil-Malmaison Cedex - France \\ 2 Laboratoire de Géophysique interne et tectonophysique, CNRS-Observatoire IRIGM, \\ BP 53X, 38041 Grenoble - France \\ e-mail : tristan.cornu@ifp.fr - frederic.schneider@ifp.fr - jean-pierre.gratier@obs-ujf.grenoble.fr
}

\begin{abstract}
Résumé - La déformation des couches géologiques en trois dimensions est une nouvelle étape dans la modélisation de bassin. Les premiers algorithmes de déformation cinématique publiés étaient des modèles « pseudo-3D », qui étaient limités à l'étude de cas construits à partir de sections topologiquement équivalentes. Le modèle cinématique proposé ici poursuit ces travaux et permet d'élargir leur domaine d'application vers un vrai traitement 3D. Pour ce faire, il utilise une approche discrète et introduit deux notions relatives à la conservation de l'aire de la surface neutre d'une couche, et à l'alternance des couches rigides et ductiles. Les résultats obtenus à l'aide du modèle sont cohérents avec ceux obtenus par les modèles pseudo-3D, et mettent en évidence la capacité de l'approche discrète à prendre en compte des géométries complexes. Il reste désormais à étendre ce modèle à des conditions à la limite plus générales que celles qui consistent à imposer un déplacement dans une seule direction donnée.

Mots-clés : cinématique 3D, modélisation de bassin, plis par flexion, restauration.
\end{abstract}

\begin{abstract}
A Discrete Kinematic Model for 3D Deformation at Sedimentary Basin Scale - The 3D deformation of a geological layer is a new step in basin modelling. The first kinematic models were "pseudo-3D" ones, and were limited to the study of cases built with topologically equivalent sections. The model detailed here follows these works, and tries to enlarge the application cases. To do this, it uses a discrete approach, and introduces two notions that deal with the area conservation of the neutral surface of each layer, and with the succession of ductile and rigid layers. The results obtained with the model are coherent with those obtained with pseudo-3D models, and they show the ability of the discrete approach to solve a problem with a complex geometry. We now have to extend the model so we can use more general boundary conditions than the one which assesses a displacement in only one direction.
\end{abstract}

Keywords: $3 D$ kinematics, basin modelling, flexural slip, restoration. 


\section{INTRODUCTION}

La restauration et la déformation des couches géologiques en trois dimensions constituent une nouvelle étape dans le domaine de la modélisation des bassins sédimentaires. Ce problème peut se traiter à l'aide d'une approche mécanique ou d'une approche cinématique. L'approche mécanique du problème a pu donner des résultats satisfaisants sur des études bidimensionnelles [1-3]. Néanmoins, une approche mécanique 3D qui intégrerait l'ensemble des paramètres géologiques propres à la modélisation de bassin n'a pas encore été réalisée. Cela est probablement dû à la complexité des phénomènes mis en jeu à l'échelle des temps géologiques et à l'absence de rhéologies adaptées [4-7]. De la même façon, un logiciel industriel du type de Udec [8,9], qui est parfaitement adapté à l'étude de blocs indépendants, implique des hypothèses trop restrictives pour des applications à des processus naturels : par exemple, la déformation doit être infinitésimale, ce qui est en contradiction avec les grandes transformations (grands déplacements et grandes déformations) que l'on observe dans la modélisation de bassin.

En raison des difficultés rencontrées au cours d'une approche mécanique, l'autre approche communément utilisée pour modéliser la déformation des bassins est l'approche cinématique. L'approche cinématique décrite ici permet de reconstituer un chemin de déformation géologiquement acceptable, prenant en compte une évolution incrémentale dans le temps, contrairement à des logiciels de dépliage du type Unfold [10] ou Patchwork [11] qui déplient instantanément. Jusqu'alors, les principaux travaux sur des modèles cinématiques ont été effectués en deux dimensions [12-19]. Par la suite, quelques modèles ont proposé des solutions «pseudo-3D ». Ils sont hélas limités à l'étude de cas cylindriques [20,21], ou construits à partir de sections 2D topologiquement équivalentes. Ces approches, si elles permettent d'appréhender un grand nombre de cas, ne peuvent pourtant rendre compte de la complexité d'un cas réellement 3D.

L'objectif n'est donc pas de fournir un modèle mécanique de déformation, mais de proposer un modèle qui soit suffisamment représentatif des processus naturels et qui puisse servir de base à des calculs de transferts (thermiques, fluides) et d'évolution des propriétés des roches (sédimentation, érosion, compaction). Afin d'obtenir une meilleure approche des géométries $3 \mathrm{D}$, le modèle proposé ici s'inspire de l'approche 2D discrète effectuée par Diviès [22]. En plus de l'approche discrète, les concepts de surface neutre et de cisaillement aux interfaces des couches ont été introduits.

\section{PRINCIPES DU MODÈLE}

La déformation d'une couche d'un bassin sédimentaire peut être considérée comme rigide-pliante [5, 23], les déformations internes étant locales et situées aux interfaces entre les couches ou aux points de rupture de pente. Cette déformation rigide-pliante est liée à deux types de mécanismes [24] :

- des processus cataclastiques peuvent permettre le mouvement de blocs par fracturation-broyage à leur limite;

- un autre mécanisme est le fluage par dissolutioncristallisation sous contrainte.

Dans ce dernier cas, les déplacements relatifs des blocs sont accommodés par des processus de dissolution, dans les zones soumises aux plus fortes contraintes (stylolites, schistosité), et de précipitation, dans les vides ouverts entre les blocs par la déformation (fentes à cristaux). Une première approche pour modéliser ce mécanisme rigide-pliant est l'utilisation de couches constituées d'éléments rigides, appelée modélisation par blocs rigides. Une représentation rigoureuse de la réalité impliquerait de considérer des blocs rigides de petite taille (mm à dm), mais la modélisation de l'ensemble d'un bassin dépasserait alors les possibilités actuelles de calcul. Le principe du modèle est donc d'utiliser des blocs de plus grande taille $(\mathrm{hm}$ à $\mathrm{km})$ en considérant que les vides et recouvrements observés sur le modèle traduisent des processus à des tailles bien inférieures dans la réalité, mais qu'ils permettent de bien modéliser les conséquences géométriques et cinématiques propres à ces déformations.

Comme le montre la figure 1, la couche est découpée en blocs (hexaèdres en 3D) qui se déplacent de façon rigide sur le support de glissement (i.e. par translation et rotation). Le volume de l'ensemble des blocs d'une couche reste constant, les blocs ayant la particularité de pouvoir s'interpénétrer ou de se dissocier, créant ainsi des vides et des recouvrements. Ainsi, le déplacement global est représenté par la somme des déplacements des blocs, tandis que la déformation est représentée par les recouvrements et les vides entre les blocs.

Il est à noter également que, pour peu que l'on puisse les estimer, des changements de volume liés par exemple à des phénomènes de compaction peuvent parfaitement être intégrés à ce processus de modélisation. Des compactions croissant avec la profondeur [22] ou bien des compactions liées à des processus tectoniques peuvent être introduites d'une manière indépendante pour chacun des blocs et à chaque incrément de déformation.

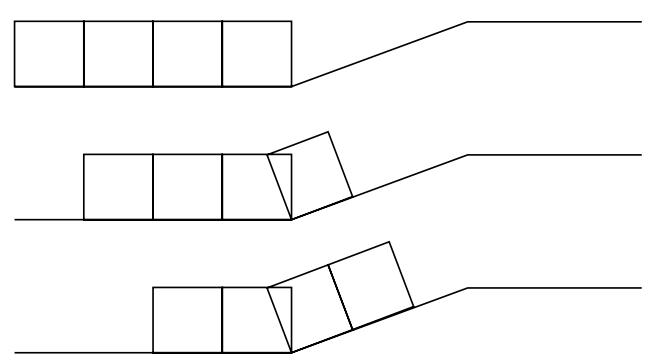

Figure 1

Mouvement de blocs rigides.

Rigid blocks motion. 
Dans la nature, la déformation d'une couche, si elle peut être considérée comme globalement rigide, comme on vient de le remarquer, est liée à un mécanisme principal, qui est un mécanisme de flexion [5, 6], dans le cas d'une région soumise à une tectonique compressive. Au cours de la flexion, la déformation de la couche a lieu préférentiellement dans la zone charnière et le long des flancs, comme le montre la figure 2.

Dans le cas de la figure 2a, le plissement entraîne une compression à l'intrados et une extension à l'extrados de la couche; l'extension et la compression sont d'autant plus importantes que la couche est épaisse. Dans le cas des figures $2 \mathrm{~b}$ et $2 \mathrm{c}$, la couche est soumise à un cisaillement parallèle à la surface de la couche, de manière continue (fig. $2 c$ ) et discontinue (fig. 2b). On remarquera la présence (fig. 2a) d'une ligne pointillée qui symbolise la ligne neutre de la couche (ou surface en 3D) : sa longueur - et par extension, sa surface dans les cas tridimensionnels - reste constante au cours de la transformation.

C'est à partir de cette remarque sur la surface neutre que le modèle exposé ici a été élaboré. L'objectif est d'utiliser la surface neutre pour modéliser la déformation. La difficulté est alors diminuée puisque l'on ne déforme plus un volume, mais des surfaces superposées dans l'espace. D'autre part, l'aspect rigide évoqué précédemment est ramené aux arêtes verticales des éléments : celles-ci conservent leur hauteur et sont donc indéformables, comme le sont les blocs. Chaque arête est reconstruite à l'aide d'une rotation autour de la surface neutre, ce qui permet soit de conserver l'épaisseur (et la taille) des blocs dans les cas les plus simples, soit de modifier cette épaisseur (et la taille) si l'on peut l'estimer, dans le cas d'une prise en compte de la compaction verticale par exemple.

Un autre problème est celui de la relation entre les couches successives. En effet, les vides et recouvrements occasionnés
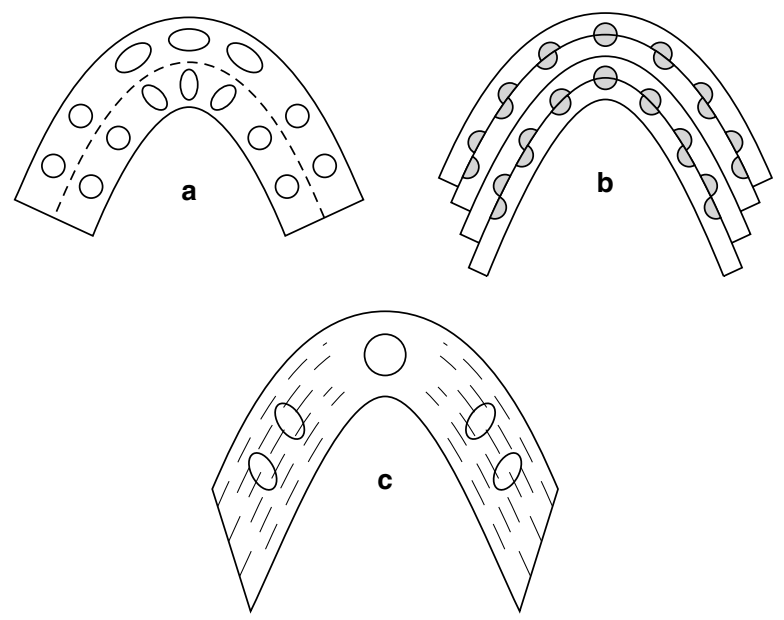

Figure 2

Mécanisme du plissement par flexion [5].

Flexural slip mechanism [5]. par le déplacement rendent difficile la définition du toit d'une couche, ce qui empêche de bien prendre en compte le glissement aux interfaces entre les couches, alors que l'hypothèse de glissement entre les couches est fondamentale dans les modèles de pli par flexion. C'est notamment le cas du mécanisme de la figure $2 \mathrm{~b}$ qui, dans le détail, est souvent associé à des plis, ou à de la schistosité d'entraînement (fig. 3). Ce problème est traité par l'ajout d'une interface cisaillante entre deux couches plissées par flexion (fig. 4). Ceci permet d'assurer dans le même temps une parfaite continuité du maillage initial des couches successives.

Le modèle comporte trois hypothèses principales. Les deux premières découlent directement des hypothèses classiques du plissement par flexion et la troisième concerne la surface neutre :

- les couches se plissent en flexion et glissent les unes par rapport aux autres ;

- la transformation peut être isopache (l'épaisseur considérée est la distance normale au support de la surface neutre), ou bien à épaisseur variable si on peut l'estimer à chaque incrément (à l'aide d'une loi de compaction par exemple [25]) ;

- l'aire de la surface neutre est conservée au mieux au cours de la transformation, ou bien modifiée si l'on peut estimer ses variations (par une quantification éventuelle de sa déformation interne).

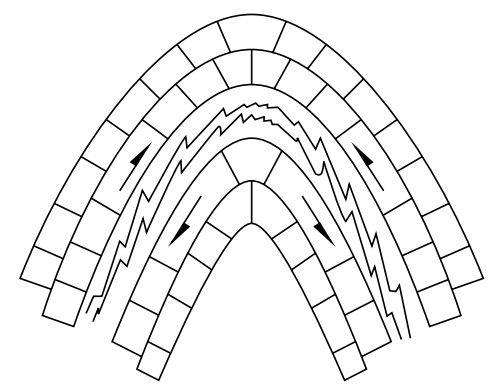

Figure 3

Plis d'« entraînement induit » [5].

Disharmonic folding during layer parallel slip [5].

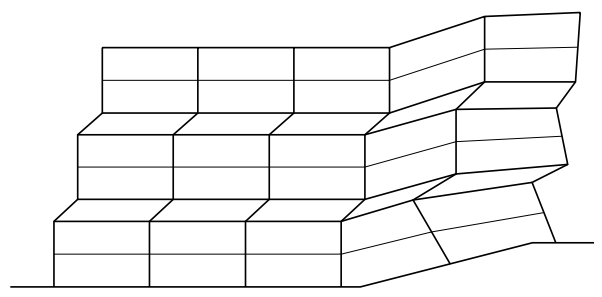

Figure 4

Interface cisaillante.

Sheared layer. 
Dans les exemples qui suivent, l'épaisseur et la surface sont conservées. La surface neutre est déformée par un glissement parallèle au support de la couche, ce qui permet à la surface neutre d'être à distance constante du support. Les arêtes verticales, qui sont reconstruites grâce à une rotation autour de la surface neutre en conservant l'angle avec le support, représentent la partie rigide de la déformation évoquée plus haut.

\section{FORMULATION MATHÉMATIQUE}

\subsection{Le domaine}

Le bassin est décomposé en trois sous-parties :

- tout d'abord en blocs séparés par des failles ;

- chaque bloc est alors subdivisé en couches;

- puis, les couches en éléments (fig. 5).

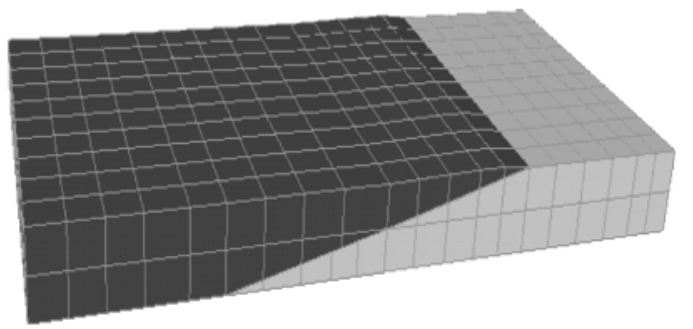

Figure 5

Vue 3D de la rampe avant déformation.

Basin 3D view before deformation.

Les éléments de la couche sont des hexaèdres à huit sommets.

Les couches vont permettre de construire le support de glissement, qui est une surface paramétrée $C^{0}$ : assemblage de surfaces planes $C^{1}$ définies à l'aide des coordonnées des sommets des éléments. Pour la première couche du bloc, le support $C^{0}$ est construit à partir des faces à la base des éléments : des quadrilatères qui, " découpés », donnent deux surfaces triangulaires $C^{1}$. Pour les couches supérieures, le même procédé est conservé, mais sur le toit de la couche précédente.

\subsection{Les inconnues}

Les inconnues de la transformation sont les coordonnées des nœuds (les sommets des éléments) après transformation. La déformation étant supposée rigide, les coordonnées de la transformée sont fournies par la solution de la translation appliquée à chaque nœud de la surface neutre :

$$
\vec{t}: \delta \vec{v}
$$

où $\delta$ est une distance de déplacement et $\vec{v}$ la direction unitaire du déplacement.

Sous forme paramétrique :

$$
\begin{gathered}
M^{\prime}=\vec{t}(M) \\
\left\{\begin{array}{l}
M_{x}^{\prime}=M_{x}+v_{x} \delta \\
M_{y}^{\prime}=M_{y}+v_{y} \delta \\
M_{z}^{\prime}=M_{z}+v_{z} \delta
\end{array}\right.
\end{gathered}
$$

\subsection{Le déplacement}

Le déplacement de la couche se fait à partir du déplacement de la surface neutre. La surface neutre est supposée dans un premier temps comme étant la surface passant par les points milieux des arêtes verticales de la couche. Son déplacement se confond avec celui de ces points milieux d'arêtes. L'opération appliquée pour la déformation étant une translation curvilinéaire (une translation sur une abscisse curviligne appartenant au support de glissement), la surface se conserve. L'autre principe de conservation à vérifier est l'isopacité des couches au cours du déplacement. Pour ce faire, le point est translaté sur une droite parallèle au support, et à distance constante du support. Le maintien de cette distance est réalisé par l'introduction des plans bissecteurs du support (l'équivalent des kinks du modèle de Contreras et Sutter [12]) et par le système d'équations paramétriques décrit ci-dessous.

Dans un premier temps, on calcule la distance du milieu d'arête $M$ avec le point d'intersection du plan médiateur $I$. Soient $\overrightarrow{v_{H}}$ la projection de $\vec{v}$ sur le plan du support $P_{S}$ suivant la normale à $P_{S}$, et $P_{\text {med }}$ le plan médiateur, tels que :

$$
\begin{gathered}
\overrightarrow{v_{H}}=\left\{\begin{array}{l}
v_{h_{x}} \\
v_{h_{y}} \\
v_{h_{z}}
\end{array}\right. \\
\left(P_{\text {med }}\right): \alpha_{m} x+\beta_{m} y+\lambda_{m} z+h=0
\end{gathered}
$$

Le point $I$ est l'intersection de $P_{\text {med }}$ avec la droite $D$ définie par :

$$
D:\left\{\begin{array}{l}
M_{x}+v_{h_{x}} t \\
M_{y}+v_{h_{y}} t \\
M_{z}+v_{h_{z}} t
\end{array}\right.
$$

Donc, les coordonnées de I s'écrivent :

$$
I:\left\{\begin{array}{l}
M_{x}+v_{h_{x}} t_{i} \\
M_{y}+v_{h_{y}} t_{i} \\
M_{z}+v_{h_{z}} t_{i}
\end{array}\right.
$$


$\operatorname{avec} t_{i}$ :

$$
t_{i}=\frac{-\left(\alpha_{m} M_{x}+\beta_{m} M_{y}+\lambda_{m} M_{z}+h\right)}{\left(\alpha_{m} v_{h_{x}}+\beta_{m} v_{h_{y}}+\lambda_{m} v_{h_{z}}\right)}
$$

Soit $d=\|\overrightarrow{M I}\|$, sachant que $\delta$ est la distance du déplacement, alors :

- si $d>\delta$, le point $M^{\prime}=\vec{t}(M)$ est défini par :

$$
M^{\prime}=\left\{\begin{array}{l}
M_{x}+v_{h_{x}} \delta \\
M_{y}+v_{h_{y}} \delta \\
M_{z}+v_{h_{z}} \delta
\end{array}\right.
$$

- si $d=\delta$, le point $M^{\prime}$ est confondu avec le point $I$;

- si $d<\delta$, le point $M$ doit encore se déplacer de la distance $\delta-d$. On effectue donc la même série d'opérations, en considérant $I$ comme nouveau point $M$ et l'on calcule le nouveau plan bissecteur $P_{S_{2}}, M$ ' est alors défini par :

$$
M^{\prime}=\left\{\begin{array}{l}
I_{x}+v_{h_{x}}(\delta-d) \\
I_{y}+v_{h_{y}}(\delta-d) \\
I_{z}+v_{h_{z}}(\delta-d)
\end{array}\right.
$$

Par commodité d'écriture, la notation de $\overrightarrow{v_{h}}$ n'a pas changé, mais le vecteur évolue en fonction du support.

\subsection{Reconstruction des arêtes}

La reconstruction des arêtes est la deuxième partie de l'algorithme de déformation. Les arêtes verticales sont supposées rigides, donc leur hauteur est conservée, mais aussi l'angle qu'elles forment avec le support. Pour réaliser ces conservations, on utilise le fait que la distance normale au support d'un point de la surface neutre est constante : pour conserver l'angle, on va supposer constante la distance entre la base de l'arête et la projection normale sur le support du point de la surface neutre appartenant à l'arête. Cette distance est symbolisée sur la figure 6 par le vecteur curvilinéaire $\vec{\mu}$.

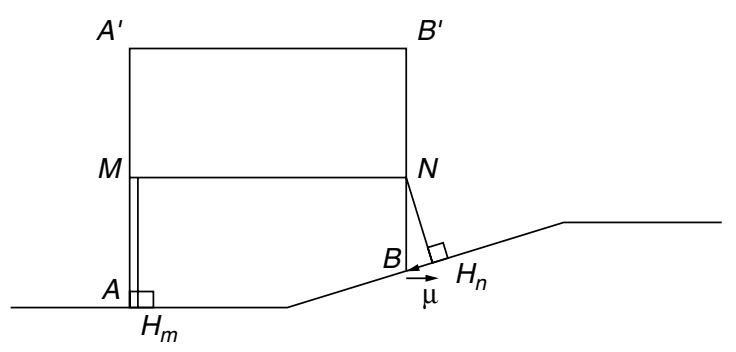

Figure 6

Principe de reconstruction de l'arête.

Edge reconstruction.
Une fois la reconstruction effectuée, les couches glissant les unes sur les autres, la connectivité du maillage initial peut être brisée, comme on peut le constater pour le modèle Foldis [22]. L'interface cisaillante qui modélise l'alternance de couches rigides et ductiles, dans le même esprit que les plis d'entraînement induits, permet de conserver cette connectivité du maillage (fig. 4). Cette interface peut être d'épaisseur nulle ou non, suivant la modélisation recherchée.

\section{RÉSULTATS PRÉLIMINAIRES}

\subsection{Pli de rampe cylindrique}

L'exemple suivant a pour but de tester la validité de l'algorithme 3D. Le cas test est une succession de dix coupes géologiques identiques ; le domaine a une longueur de $20 \mathrm{~km}$, une largeur de $10 \mathrm{~km}$ et une hauteur de $2 \mathrm{~km}$. Le bassin est composé de deux blocs, les couches du bloc mobile sont soumises à trois déplacements successifs de $1000 \mathrm{~m}$ (fig. 7 et 8). La quantité de déplacement est appliquée de manière homogène sur le bord, dans la direction parallèle à celle de la rampe.

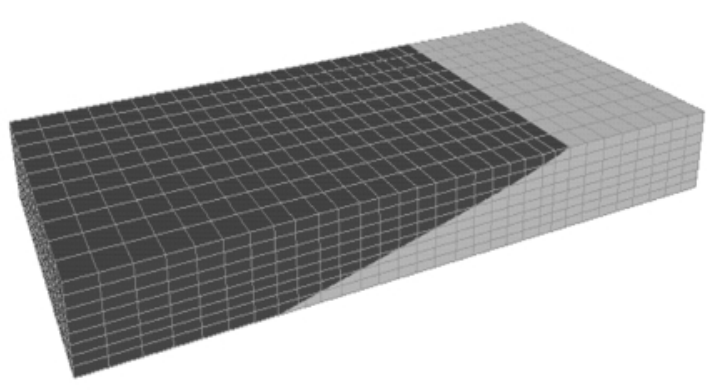

Figure 7

Vue 3D de la rampe avant déformation.

Thrust $3 D$ view before deformation.

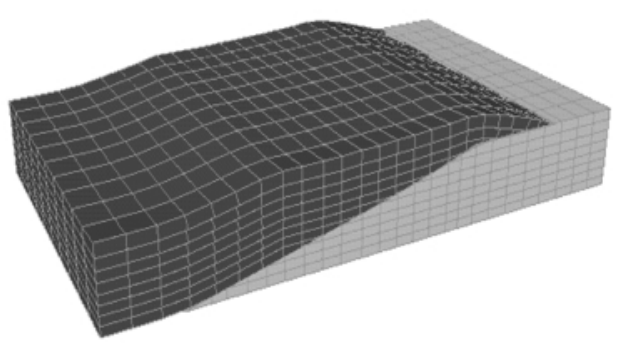

Figure 8

Vue 3D de la rampe après déformation.

Thrust $3 D$ view after deformation. 

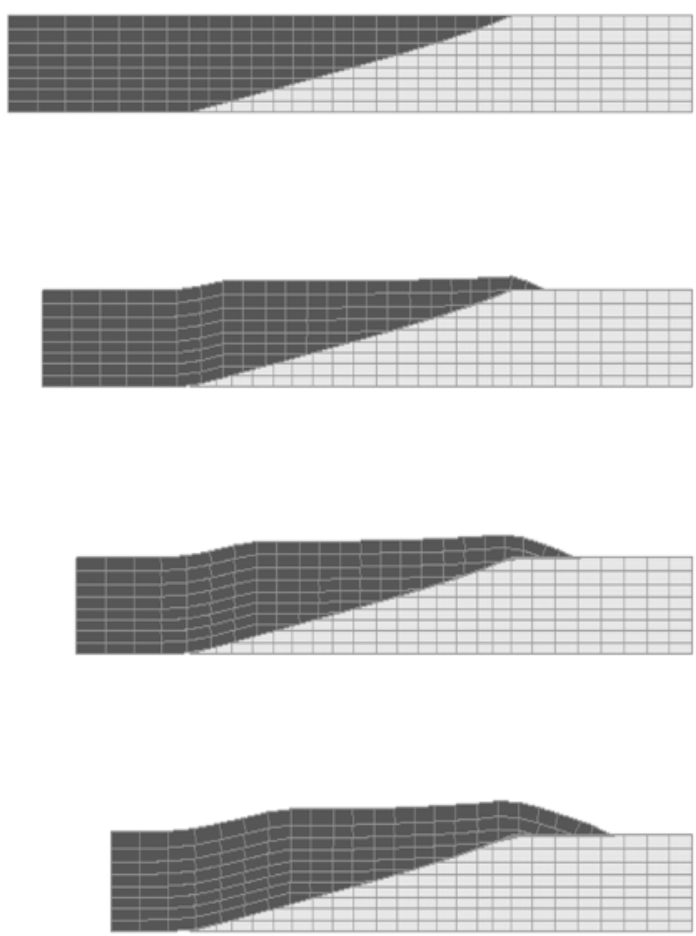

Figure 9

Évolution du bassin en section 2D.

$2 D$ evolution of the thrust.

La figure 9 représente l'évolution d'une section du bassin à chaque incrément de temps. On constate que les résultats obtenus sont similaires à ceux fournis par des codes bidimensionnels classiques.

\subsection{Pli de rampe avec cisaillement}

Le bassin précédent (composé de quatre couches cette foisci) est déformé à l'aide d'un cisaillement parallèle à la direction de la rampe, tel que la distance de déplacement $\delta$ soit définie, à chaque incrément, par :

$$
\delta=1000 \frac{\left(y_{\max }-y\right)}{y_{\max }}
$$

Le bassin aura donc un bord fixe, tandis que le bord opposé se déplacera de $3000 \mathrm{~m}$ (fig. 10). De nouveau, les résultats sont cohérents par rapport aux travaux effectués auparavant pour les modèles semi-3D [21].

\subsection{Pli de rampe variable}

Le bassin considéré n'est plus cylindrique : le pendage de la rampe évolue dans la largeur, pour passer de 20 à plus de $60^{\circ}$. La condition à la limite est un déplacement uniforme : $1000 \mathrm{~m}$ sur un pas de temps (fig. 11 et 12).
Les vues en section sont illustrées en figures 13 et 14 . Les résultats obtenus sont une fois de plus cohérents et similaires à ceux proposés par des méthodes géométriques semi-3D. Néanmoins, le pendage très fort de la rampe (supérieur à $60^{\circ}$ ) est un cas que les modèles classiques ne peuvent résoudre. L'algorithme proposé ici permet donc l'étude de cas à la géométrie plus complexe.

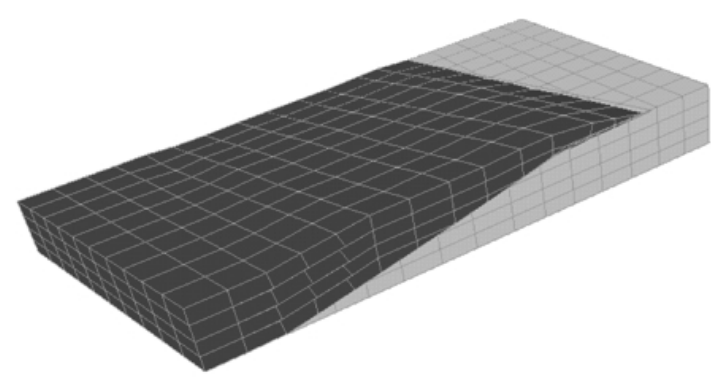

Figure 10

Vue $3 \mathrm{D}$ de la rampe après déformation avec cisaillement. $3 D$ view of the sheared thrust.

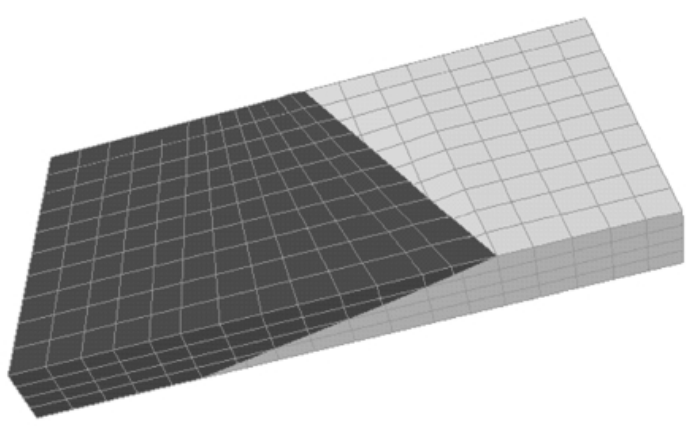

Figure 11

Vue 3D de la rampe variable avant déformation. $3 D$ view before deformation of the thrust with variable slope.

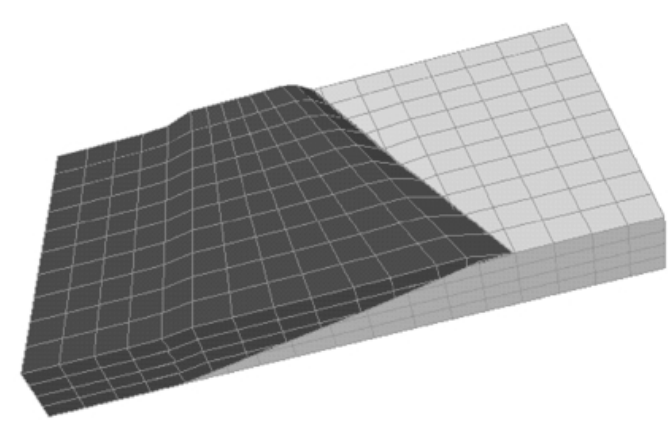

Figure 12

Vue 3D de la rampe variable après déformation.

$3 D$ view after deformation of the thrust with variable slope. 

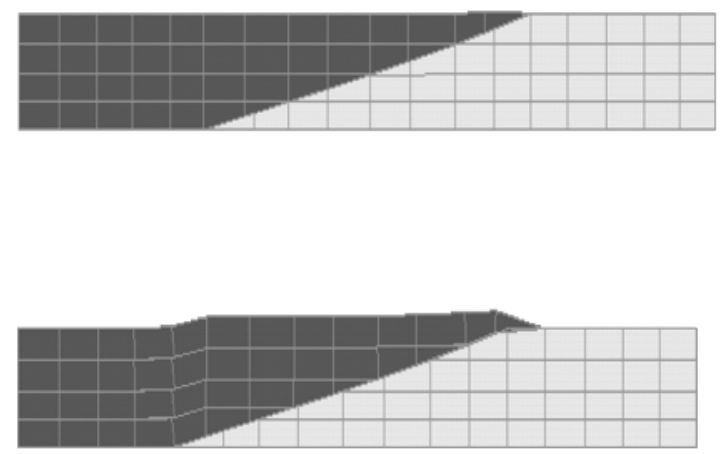

Figure 13

Évolution des sections de la rampe à $20^{\circ}$.

$2 \mathrm{D}$ evolution of the $20^{\circ}$ slope.
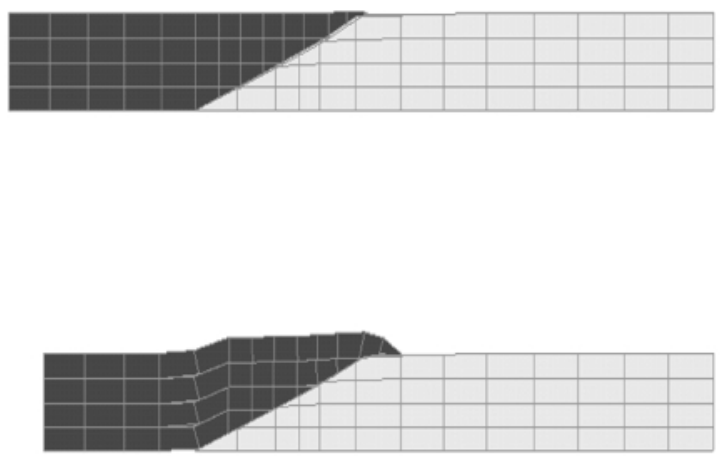

Figure 14

Évolution des sections de la rampe à $60^{\circ}$.

Evolution of the $60^{\circ}$ slope.

\section{CONCLUSION}

Le modèle proposé s'inscrit dans le prolongement des travaux effectués en modélisation de bassin [26, 27]. Il a pour objectif de permettre l'introduction de cinématiques plus complexes qui prennent en compte les failles et les glissements couche à couche. Ce modèle présente l'avantage d'être simple dans ses hypothèses, ce qui le rend simple à comprendre et à implémenter. Sa simplicité ne l'empêche pas d'introduire deux concepts qui, s'ils étaient connus des géologues, n'avaient encore jamais été utilisés de manière explicite : la conservation de l'aire de la surface neutre, et l'alternance de couches rigides et ductiles. Ces deux hypothèses permettent pour l'une de mieux approcher les déformations internes subies par une couche, pour l'autre de mieux représenter le cisaillement que l'on peut observer entre deux couches rigides.

Les résultats présentés avec le modèle montrent sa capacité à traiter l'ensemble des problèmes que peut traiter un modèle semi-3D, dans la mesure où il est limité à l'étude de bassins constitués de sections topologiquement équivalentes, même si l'approche discrète permet de traiter des cas complexes. D'autre part, contrairement à un modèle tel que celui proposé par l'université de Keele [28], qui est direct en cisaillement vertical et inverse à l'aide d'un algorithme de dépliage de type Unfold, le modèle décrit ici est totalement réversible et offre la possibilité de s'intégrer dans un logiciel de modélisation de bassin. La suite des travaux envisagés est la généralisation du modèle pour pouvoir prendre en compte des conditions aux limites plus générales, afin de traiter de déformations complètement tridimensionnelles.

\section{RÉFÉRENCES}

1 Barnichon, J.D. (1998) Finite Element Modelling in Structural and Petroleum Geology. Thèse, université de Liège.
2 Niño, F., Chéry, J. et Gratier, J.P. (1998) Mechanical Modelling of the Ventura Basin: Origin of the San Cayetano Thrust Fault and Interaction with the Oak Ridge Fault. Tectonics, 17, 955-972.

3 Hassani, R. (1994) Modélisation numérique de la déformation des systèmes géologiques. Thèse, université de Montpellier II.

4 Charlez, P.A. (1991) Rock Mechanics, Theoretical Fundamentals, vol. 1, Éd. Technip, Paris.

5 Nicolas, A. (1984) Principes de tectonique, Masson, Paris.

6 Ramsay, J.G. et Huber, M.I. (1987) The Techniques of Modern Structural Geology—Folds and Fractures, vol. 2, Academic Press.

7 Lamoureux-Var, V. (1997) Modélisation de la compaction dans les bassins sédimentaires, approche mécanique. Thèse, École polytechnique.

8 Cundall, P.A. (1998) Formulation of a Three-Dimensional Distinct Element Model, Part 1: A Scheme to Detect and Represent Contacts in a System Composed of Many Polyhedral Blocks. Int. J. Rock Mech. Min. Sci. \& Geomech. Abs., 25, 3, 107-116.

9 Hart, R., Cundall., P.A. et Lemos, J. (1998) Formulation of a Three-Dimensional Distinct Element Model, Part II: Mechanical Calculations for Motion and Interaction of a System Composed of Many Polyhedral Blocks. Int. J. Rock Mech. Min. Sci. \& Geomech. Abs., 25, 3, 117-125.

10 Gratier, J.P. et Guillier, B. (1993) Compatibility Constraints on Folded and Faulted Strata and Calculation of Total Displacement using Computational Restoration (Unfold Program). Journal of Structural Geology, 15, 3-5, 391-402.

11 Bennis, C., Vézien, J.M. et Iglésias, G. (1991) Piecewise Surface Flattening for Non-Distorted Texture Mapping. Computer Graphics, 25, 4, 237-246.

12 Contreras, J. et Suter, M. (1990) Kinematic Modelling of Cross-Sectional Deformation Sequences by Computer Simulation. Journal of Geophysical Research, 95, 2191321929.

13 Dahlstrom, C.D.A. (1969) Balanced Cross Sections. Canadian Journal of Earth Sciences, 6, 743-757.

14 De Paor, D.G. (1988) Balanced Section in Thrust Belts, Part 1: Construction. The American Association of Petroleum Geologist Bulletin, 72, 1, 73-90.

15 De Paor, D.G. (1988) Balanced Section in Thrust Belts, Part 2: Computerized Line and Area Balancing. Geobyte, mai, 33-37. 
16 Suppe, J. (1983) Geometry and Kinematic of Fauld-Bond Folding. American Journal of Science, 283, 684-721.

17 Waltham, D. (1989) Finite Difference Modelling of Hangingwall Deformation. Journal of Structural Geology, 11, 433-437.

18 Waltham, D. (1990) Finite Difference Modelling of Sandbox Analogues, Compaction and Detachment Free Deformation. Journal of Structural Geology, 12, 375-381.

19 Zoetemeijer, R. (1992) Tectonic Modelling of Forelands Basins, Thin Skinned Thrusting, Syntectonic Sedimentation and Lithospheric Flexure. Thèse, Vrije Universiteit Amsterdam.

20 Shaw, J.H., Hook, S.C. et Suppe, J. (1994) Structural Trend Analysis by Axial Surface Mapping. AAPG Bulletin, 78, 700721.

21 Scott Wilkerson, M. et Medwedeff, D.A. (1991) Geometrical Modeling of Fault-Related Folds: A Pseudo-ThreeDimensional Approach. Journal of Structural Geology, 13, 801-812.

22 Diviès, R. (1997) Foldis : un modèle cinématique de bassins sédimentaires par éléments discrets associant plis, failles, érosion/sédimentation et compaction. Thèse, université Joseph-Fourier de Grenoble.
23 Merle, O. (1984) Déplacement et déformation des nappes superficielles. Revue de géologie dynamique et géographie physique, 25, 3-17.

24 Gratier, J.P. (1993) Le fluage des roches par dissolutioncristallisation sous contrainte, dans la croûte supérieure. Bulletin de la société géologique de France, 164, 2, 267-287.

25 Schneider, F., Potdevin, J.L. et Wolf, S. (1996) Mechanical and Chemical Compaction Model for Sedimentary Basin Simulators. Tectonophysics, 263, 307-317.

26 Schneider, F. et Wolf, S. (2000) Quantitative HC Potentiel Evaluation using 3D Basin Modelling: Application to Franklin Structure, Central Graben, North Sea, UK. Marine and Petroleum Geology (in press).

27 Schneider, F., Wolf, S., Faille, I. et Pot, D. (2000) A 3D Basin Model for Hydrocarbon Potential Basin Evaluation: Application to Congo Offshore. Oil and Gas Science \& Technology, 55, 1, 3-13.

28 Egan, S., Kane, S., Buddin, T., Williams, G. and Hadgetts, D. (1999) Computer Modelling and Visualisation of the Structural Deformation Caused by Movement along Geological Faults. Computers and Geosciences, 25, 283-297.

Manuscrit définitif reçu en novembre 1999 